\title{
A Non-Demolition Photon Counting Method by Four-Level Inverted Y-Type Atom
}

\author{
Siamak Khademi*, Samira Alipour \\ Department of Physics, University of Zanjan, Zanjan, Iran. \\ *Corresponding Author's Email: khademi@ znu.ac.ir
}

Received: Apr. 2, 2016, Revised: Aug. 20, 2016, Accepted: Sep. 26, 2016, Available Online: Feb. 18, 2017

DOI: 10.18869 /acadpub.ijop.11.1.63

\begin{abstract}
The semi-classical model of atomfield interaction has been fully studied for some multilevel atoms, e.g. Vee, $\Lambda$, Cascade $\Xi, Y$, and inverted $Y$ and so on. This issue is developed into the full-quantum electrodynamics formalism, where the probe and coupling electromagnetic fields are quantized. In this article, we investigate the full-quantum model of absorption and dispersion spectrum of trapped four-levels inverted $Y$ type atoms, interacting with a probe beam of photons as well as two-mode trapped coupling photons. It is shown that the measurement of the maximum of absorption of the probe field and its detuning gives us simply the number of two-mode coupling photons, individually. An experimental setup for this non-demolition photon counting method is proposed and the numbers of coupling photons are obtained analytically.
\end{abstract}

KEYWORDS: absorption, electromagnetically induced transparency, full-quantum model, non-demolition photon counting, semiclassical model.

\section{I.INTRODUCTION}

Evaluation and manipulation of photonic and atomic states in quantum cavities are an attractive topic for many authors and have many applications in quantum optics [1-6], quantum memory [7-9], quantum information $[10,11]$, and quantum computation [12, 13]. The semi-classical and full-quantum model of multilevel atoms, interacting with single or multi-mode electromagnetic fields, are fully studied experimentally and theoretically [1417], in linear and non-linear [13, 18, 19] mediums. These studies have found many applications, e.g. in electromagnetically induced transparency [20-22], electromagnetically induced absorption [2324], laser without inversion [25, 26], AutlerTownes effect [27-29], sub and super-luminal effects [30-32], optical bistability [33], entanglement [34], photons counting [3-6] and so on.

It is known that the absorption and dispersion spectra of multilevel atoms, interacting with multi-modes coupling and probe fields, contain much information about the properties of coupling fields as well as the optical properties of atoms [3-6]. In the present article, we show that the number of trapped coupling photons (which are microscopic quantities) could be obtained from the absorption spectrum of the probe field (which is a macroscopic quantity), analytically. Our photon counting method is also among the non-demolition measurement methods. In this article, we focus on the absorption spectrum of an ensemble of cold inverted Y multilevel atoms which are trapped in a high quality cavity electrodynamics. These multilevel atoms are interacted with two mode coupling photons which are trapped in another (or the same) cavity. A probe beam of photons is injected into the cold ensemble of multi-level atoms and the amount of its absorption spectrum, in terms of its detuning, is measured by a high efficiency single photon detector. It is shown that the maximum amount of absorptions and their detunings are strongly affected by the number of trapped coupling photons. 
The article is organized as follows: In Section two, four different profiles of absorption spectra for inverted Y-type atoms are investigated and analyzed. Their master's equations are obtained in a rotating frame and steady state, and are also solved in weak field approximation. Section three is dealing with a photon counting method corresponding to different profile's properties of absorption spectra. Numbers of coupling photons are analytically derived from the difference between the detunings of absorption peaks. Section four is also devoted to the conclusions.

\section{FOUR-LEVEL INVERTED Y-TYPE ATOM INTERACTED WITH THREE MODES OF PHOTONS}

In this work, an inverted Y-type (IYT) configuration atom, which has been experimentally realized in $\mathrm{Rb}$ atoms, interacting with two coupling trapped photons as well as a probe beam of photons is investigated. The states of atoms are denoted by $|j\rangle$ where $j=a, b, c, d$ (see Fig. 1). The state, frequency and detuning of the probe beam of photons (and two coupling photons) are also denoted by $\left|n_{1}\right\rangle$ (and $\left|n_{2}\right\rangle$ and $\left|n_{3}\right\rangle$ ), $\left|v_{1}\right\rangle$ (and $\left|v_{2}\right\rangle$ and $\left|v_{3}\right\rangle$ ), and $\Delta_{1}$ (and $\Delta_{2}$ and $\Delta_{3}$ ), respectively. All modes of photons are coupled, near-resonantly, with two corresponding states of atom as shown in Fig. 1. Each mode of coupling photons is also assumed to be trapped into an individual high quality (high Q-factor) quantum cavity, which is on resonance with the corresponding frequency of photons (see Fig. 2).

Furthermore, an ensemble of IYT atoms is also trapped in another cavity which is perpendicular to the cavities shown in Fig. 2 . The probe photons are also injected into the ensembles of atoms, one by one, and a detector measures their absorption spectrum. The interaction Hamiltonian of the system in the interaction picture is given by the JaynesCumming model $[16,17]$.

$$
\begin{aligned}
\hat{V}= & -\hbar g_{1}\left(\sigma_{b a} \hat{a}_{1} e^{i \Delta_{1} t}+\sigma_{a b} \hat{a}_{1}^{\dagger} e^{-i \Delta_{1} t}\right) \\
& -\hbar g_{2}\left(\sigma_{c b} \hat{a}_{2} e^{i \Delta_{2} t}+\sigma_{b c} \hat{a}_{2}^{\dagger} e^{-i \Delta_{2} t}\right) \\
& -\hbar g_{3}\left(\sigma_{b d} \hat{a}_{3} e^{i \Delta_{3} t}+\sigma_{d b} \hat{a}_{3}^{\dagger} e^{-i \Delta_{3} t}\right)
\end{aligned}
$$

where $\hat{a}_{1}, \hat{a}_{2}$, and $\hat{a}_{3}\left(\hat{a}_{1}^{\dagger}, \hat{a}_{2}^{\dagger}\right.$ and $\left.\hat{a}_{3}^{\dagger}\right)$ are the annihilation (creation) operators for the probe and two coupling photons, respectively. The couplings constants are given by $g_{k}=\overrightarrow{\mathrm{P}}_{\mathrm{ij}} \cdot \hat{\varepsilon}_{k} E_{k} / \hbar,(k=1,2,3, j=a, b, c, d)$ and $E_{k}=\left(\hbar v_{k} / 2 \varepsilon_{0} v\right)^{1 / 2}$, is the amplitudes of the $k$-th mode of the electric field with the polarization direction $\hat{\varepsilon}_{k}$ in the cavity volume $v$. The operator $\sigma_{i j}=|i\rangle\langle j|$, is the transition operator from the atomic states $|j\rangle$ to $|i\rangle$, and $\Delta_{1}=\omega_{a b}-v_{1} \quad\left(\Delta_{2}=\omega_{b c}-v_{2}, \Delta_{3}=\omega_{d b}-v_{3}\right)$, $\omega_{a b}\left(\omega_{b c}, \omega_{d b}\right)$ and $\overrightarrow{\mathrm{P}}_{\mathrm{ij}}=e\langle i|\vec{r}| j\rangle$ are detuning, atomic transition frequency and dipole moment vector corresponding to the transition $|i\rangle \rightarrow|j\rangle$, respectively.

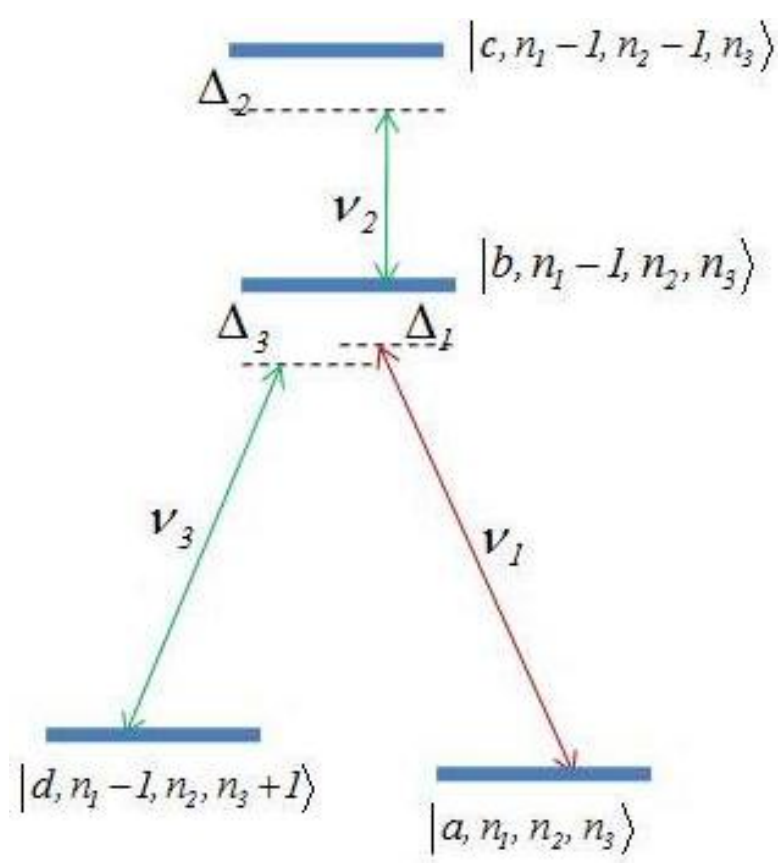

Fig. 1. Four-level IYT atomic system. The probe photon transition is shown by a red arrow. The atom-photon state is initially $\left|a, n_{1}, n_{2}, n_{3}\right\rangle$. The upper states spontaneously decay into the lower states. In this case, the decay rates for the transitions $|b\rangle \rightarrow|a\rangle,|c\rangle \rightarrow|b\rangle,|b\rangle \rightarrow d$, and $|d\rangle \rightarrow|a\rangle$ are $2 \gamma_{1}, 2 \gamma_{2}, 2 \gamma_{3}$, and $2 \gamma_{4}$ 


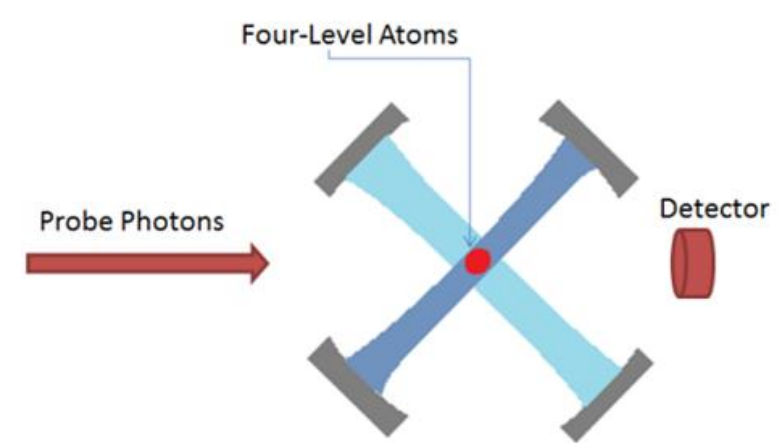

Fig. 2. Two modes coupling photons are trapped into two individual high quality quantum cavities, each assumed to be on resonance with its coupling mode frequency. The red spot is a pure ensemble of four-level IYT atoms trapped in another quantum cavity (which is not shown and may be perpendicular to the others). The probe photons are injected into the ensemble of atoms and their absorption spectrum is measured by a detector.

In the absence of the probe beam, the trapped coupling photons $n_{2}$ and $n_{3}$ are simultaneously interacted with the IYT atoms. In the steady state and weak field approximation, the state $|a\rangle$ is populated and the populations of other states are vanished. Thus the total atom-photon state is initially (before the injection of the probe beam photons) at $\left|a, 0, n_{2}, n_{3}\right\rangle$ state. By applying the rotating wave approximation, the dynamical equations of the density operator for the coherence terms

$$
\begin{aligned}
\dot{\tilde{\rho}}_{b a}= & -\left[\gamma_{1}+\gamma_{3}+i \Delta_{1}\right] \tilde{\rho}_{b a}+i g_{1} \sqrt{n_{1}}\left[\tilde{\rho}_{a a}-\tilde{\rho}_{b b}\right]+ \\
& i g_{2} \sqrt{n_{2}+1} \tilde{\rho}_{c a}+i g_{3} \sqrt{n_{3}+1} \tilde{\rho}_{d a}, \\
\dot{\tilde{\rho}}_{c a}= & -\left[\gamma_{2}+i\left(\Delta_{1}+\Delta_{2}\right)\right] \tilde{\rho}_{c a}-i g_{1} \sqrt{n_{1}} \tilde{\rho}_{c b}+i g_{2} \sqrt{n_{2}} \tilde{\rho}_{b a}, \\
\dot{\tilde{\rho}}_{d a}= & -\left[\gamma_{4}+i\left(\Delta_{1}-\Delta_{3}\right)\right] \tilde{\rho}_{d a}-i g_{1} \sqrt{n_{1}} \tilde{\rho}_{d b}+ \\
& i g_{3} \sqrt{n_{3}+1} \tilde{\rho}_{b a}
\end{aligned}
$$

are obtained from the master equation: $\frac{d \hat{\rho}}{d t}=\frac{-i}{\hbar}[\hat{V}, \hat{\rho}]+L(\hat{\rho})$,

where $\hat{\rho}=|\psi(t)\rangle\langle\psi(t)|$ is the density operator, $L(\hat{\rho})=\frac{\gamma}{2}\left[2 \sigma_{-} \hat{\rho} \sigma_{+}-\sigma_{+} \sigma_{-} \hat{\rho}-\hat{\rho} \sigma_{+} \sigma_{-}\right]$is

Lindblad decay term [17] and the general atom-photon state

$$
\begin{aligned}
|\psi(t)\rangle & =C_{a}(t)\left|a, n_{1}, n_{2}, n_{3}\right\rangle \\
& +C_{b}\left|b, n_{1}-1, n_{2}, n_{3}\right\rangle \\
& +\mathrm{C}_{\mathrm{c}}\left|c, n_{1}-1, n_{2}, n_{3}+1\right\rangle \\
& +\mathrm{C}_{\mathrm{d}}\left|d, n_{1}-1, n_{2}-1, n_{3}+1\right\rangle .
\end{aligned}
$$

In this case, the decay rates for the transitions $|b\rangle \rightarrow|a\rangle, \quad|c\rangle \rightarrow|b\rangle, \quad|b\rangle \rightarrow|d\rangle, \quad$ and $|d\rangle \rightarrow|a\rangle$ are assumed to be $2 \gamma_{1}, 2 \gamma_{2}, 2 \gamma_{3}$ and $2 \gamma_{4}$, respectively.

$$
\tilde{\rho}_{b a}{ }^{(1)}=\frac{i g_{1} \sqrt{n_{1}}}{\left[\gamma_{1}+\gamma_{3}+i \Delta_{1}\right]+\frac{\left(n_{3}+1\right) g_{3}^{2}}{\left[\gamma_{4}+i\left(\Delta_{1}-\Delta_{3}\right)\right]}+\frac{n_{2} g_{2}^{2}}{\left[\gamma_{2}+i\left(\Delta_{1}+\Delta_{2}\right)\right]}},
$$

The coherence term is obtained in the steady state, where the weak field approximation $\rho_{b a} \approx \tilde{\rho}_{b a}{ }^{(0)}+g_{1} \tilde{\rho}_{b a}{ }^{(1)}$ is assumed and nonperturbed density matrix elements are set to be

$\rho_{a a}{ }^{(0)}=1$,

$$
\rho_{b b}{ }^{(0)}=\rho_{c c}{ }^{(0)}=\rho_{d d}{ }^{(0)}=\rho_{b d}{ }^{(0)}=\rho_{c b}{ }^{(0)}=0 .
$$

By applying Eq. (6) in the polarizations $P_{1}=\varepsilon_{0} \chi_{a b} E_{1} \quad$ and $\quad P_{1}=2 \tilde{\rho}_{a b} g_{1}$, the susceptibility $\left(\chi_{a b}\right)$ of the atoms experienced by the probe beam of photons is obtained as

$$
\chi_{b a}=\frac{N_{a} \wp_{b a}^{2}}{\hbar \varepsilon_{0}} \frac{i}{\left[\gamma_{1}+\gamma_{3}+i \Delta_{1}\right]+\frac{\left(n_{3}+1\right) g_{3}^{2}}{\left[\gamma_{4}+i\left(\Delta_{1}-\Delta_{3}\right)\right]}+\frac{n_{2} g_{2}^{2}}{\left[\gamma_{2}+i\left(\Delta_{1}+\Delta_{2}\right)\right]}} .
$$


where $N_{a}$ is the density number of atoms and $E_{1}=\left(2 \hbar v_{1} n_{1} / \varepsilon_{0} v\right)^{1 / 2}$ is the amplitude of the quantized probe field [17]. Therefore, the real $\left(\chi^{\prime}\right)$ and imaginary $\left(\chi^{\prime \prime}\right)$ parts of the susceptibility (8) are obtained as:

$$
\begin{aligned}
\chi_{b a}^{\prime}= & \frac{1}{A^{2}+B^{2}} \times\left(\Delta_{1}+\Delta_{13} L_{3}\left(n_{3}, \Delta_{13}\right)+\right. \\
& \left.\Delta_{12} L_{2}\left(n_{2}, \Delta_{12}\right)\right),
\end{aligned}
$$

and

$$
\begin{aligned}
\chi_{b a}^{\prime \prime}= & \frac{1}{A^{2}+B^{2}} \times\left(\gamma_{1}+\gamma_{3}+\gamma_{4} L_{3}\left(n_{3}, \Delta_{13}\right)+\right. \\
& \left.\gamma_{2} L_{2}\left(n_{2}, \Delta_{12}\right)\right) .
\end{aligned}
$$

where $L_{3}\left(n_{3}, \Delta_{13}\right)=g_{3}^{2}\left(n_{3}+1\right) /\left(\gamma_{4}^{2}+\Delta_{13}{ }^{2}\right)$, $L_{2}\left(n_{2}, \Delta_{12}\right)=n_{2} g_{2}^{2} /\left(\gamma_{2}^{2}+\Delta_{12}^{2}\right)$,

$A=\gamma_{1}+\gamma_{3}+\gamma_{4} L_{3}\left(n_{3}, \Delta_{13}\right)+\gamma_{2} L_{2}\left(n_{2}, \Delta_{12}\right)$,

$B=\Delta_{1}+\Delta_{13} L_{3}\left(n_{3}, \Delta_{13}\right)-\Delta_{12} L_{2}\left(n_{2}, \Delta_{12}\right)$,

and $\Delta_{13}=\Delta_{3}-\Delta_{1}, \quad \Delta_{12}=\Delta_{1}+\Delta_{2}$. Now we focus on the absorption spectrum of the probe beam and without loss of generality, a symmetric set of parameters, $\gamma_{1}=\gamma_{2}=\gamma_{3}=0.2 \gamma, \quad \Delta_{2}=\Delta_{3}=2.5 \gamma$, $g_{2}=g_{3}=2.5 \gamma$ and $\gamma_{4}=5 \times 10^{-3} \gamma$ are chosen $(\gamma=60 \pi \mathrm{MHz})$. These values have been chosen based on the experimentally feasible parameters reported in [34-36].

The absorption spectrum has a triplet peak profile, which is plotted in Fig. 3. A fine asymmetry profile is found in the absorption spectrum, which is due to the different roles of two coupling photons $n_{2}$ and $n_{3}$, in the susceptibility (8), which is due to the effect of vacuum mode. If the coupling field $n_{3}\left(n_{2}\right)$ is turned off, its corresponding term in denominator of Eq. (8) is not (is) totally vanished. Therefore, the vacuum fluctuation field corresponding to the $3 \mathrm{rd}$ (2nd) mode of coupling field is (is not) interacted with the IYT atom.

Now, the profiles of absorption spectra are categorized in four cases:
None of coupling photons are in the vacuum state (Fig. 3).

The 3rd mode of the coupling photon $\left|n_{3}\right\rangle$ is in the non-vacuum and the 2 nd mode $\left|n_{2}=0\right\rangle$ is in the vacuum state (Fig. 4).

The 3rd mode of the coupling photon $\left|n_{3}=0\right\rangle$ is in the vacuum and the 2 nd mode $\left|n_{2}\right\rangle$ is in the non-vacuum state (dotted plot in Fig. 5).

All modes of the coupling photon states, $\left|n_{3}=0\right\rangle$ and $\left|n_{2}=0\right\rangle$, are in the vacuum state (Fig. 6).

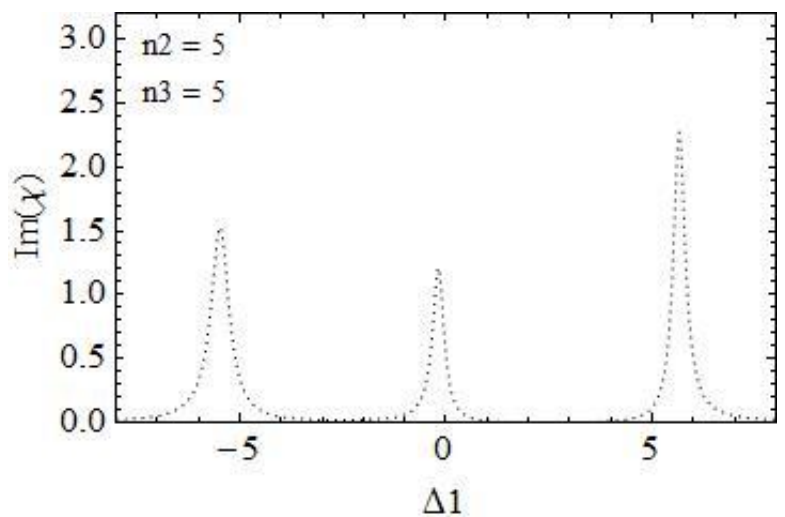

Fig. 3. Absorption spectrum of probe field versus detuning for an IYT atomic system in $n_{2}=5, n_{3}=5, \gamma_{1}=\gamma_{2}=\gamma_{3}=0.2 \gamma, \gamma_{4}=5 \times 10^{-4} \gamma$, $\Delta_{2}=\Delta_{3}=2.5 \gamma, g_{2}=g_{3}=1.5 \gamma$.

\section{1) Case a}

In this case, the absorption spectrum of the IYT atom contains a triplet absorption peak. The amount of absorption peaks and their corresponding detuning are depended on the number of coupling photons $n_{2}$ and $n_{3}$. Furthermore, in spite of the symmetric selection of parameters, Fig. 3 shows a fine asymmetric profile spectrum due to different roles of (8) for the 3nd and 3rd modes of photons and the atomic level configuration. In Section 3, we present a non-demolition method for the measurement of coupling photons in terms of the amount of absorption's peak's detuning. 


\section{2) Case b}

We have expected that, in a semi-classical model of atom-field interaction and in the absence of the coupling field $v_{2}$, a four-level IYT atom has been reduced to a three-level $\Lambda$-Type atom. Correspondingly, Fig. 4 shows the absorption spectrum profile of an IYT atom which has been reduced to the absorption profile of a three-level $\Lambda$-Type atom, even in a full-quantum model of atom-field interaction. In this case, the population of the upper level $|c\rangle$ vanishes in the steady state; therefore, the upper level $|c\rangle$ has no interaction with the vacuum fluctuation field of $v_{2}$ mode.

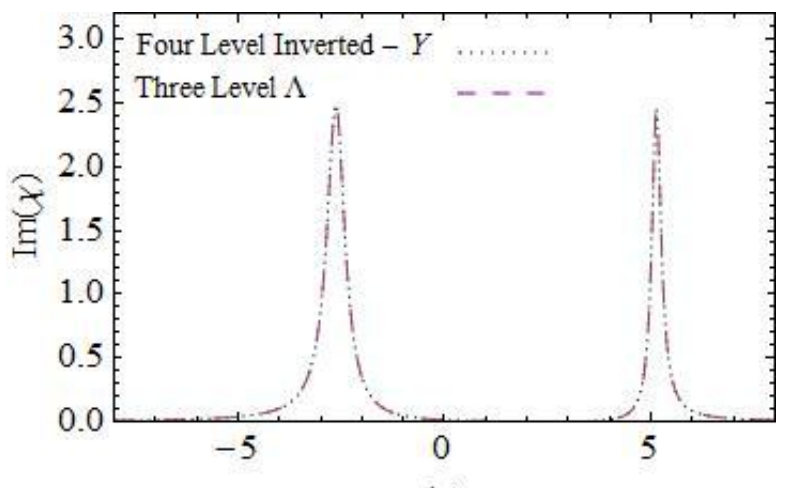

$\Delta 1$

Fig. 4. Absorption spectra of probe field versus detuning of an IYT atomic system in $n_{2}=0, n_{3}=5$, and three level $\Lambda n_{3}=5$. The parameters are similar to those chosen in Fig. 3.

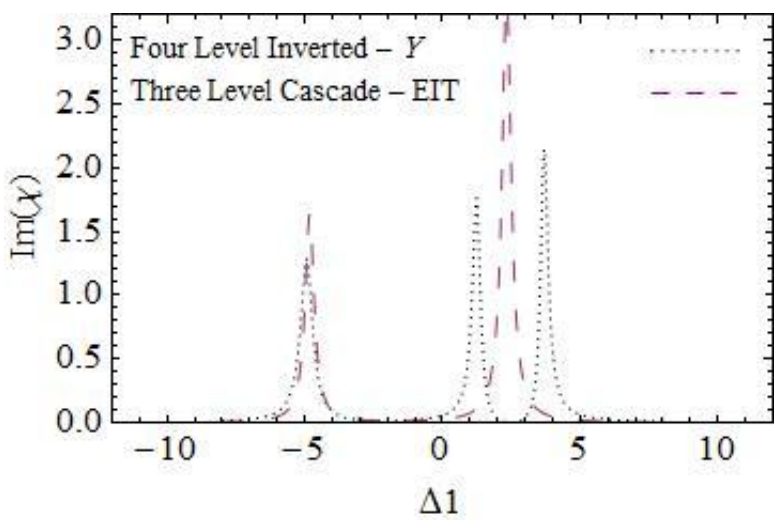

Fig. 5. Absorption spectra of probe field versus detuning for an IYT atomic system (Dotted curve) in $n_{2}=5, n_{3}=0$, and three level Cascade EIT (Dashed curve) $n_{2}=5$, The parameters are similar to those chosen in Fig. 3.

Figure 4 shows that the absorption spectrum profile depicted in Fig. 3 is exactly reduced to the absorption spectrum of a three-level $\Lambda$-type atom, where $n_{2}=0$.

\section{3) Case c}

We have also expected that, in a semi-classical model of atom-field interaction and in the absence of the coupling field $v_{3}$, a four-level IYT atom has been reduced to a three-level upper-coupled-cascade atom. But, in spite of the case $b$, where in a full-quantum model, the coupling photon $\left|n_{3}\right\rangle$ is in the vacuum state, the absorption spectrum profile of the IYT atom has not been exactly reduced to a threelevel upper-coupled-cascade atom. Figure 5 shows this difference in the profiles of the absorption spectra of these systems. This difference is due to an interaction between the atoms and vacuum fluctuations field of $v_{3}$ mode. So the second term in the denominator of Eq. (8) does not totally vanish, even where $n_{3}=0$.

\section{4) Case d}

In the absence of all coupling fields, Fig. 6 shows the absorption spectrum profile of the four-level IYT atom to contain only a double peak. In the semi-classical model of atom-field interaction, we expected that a four-level IYT atom was reduced to a simple two-level atom interacting with a single-mode field and only with a single peak in the absorption spectrum profile. As indicated in the previous cases, this difference is also due to this fact that the fourlevel IYT atoms are interacted with the vacuum fluctuation of 3 rd coupling field.

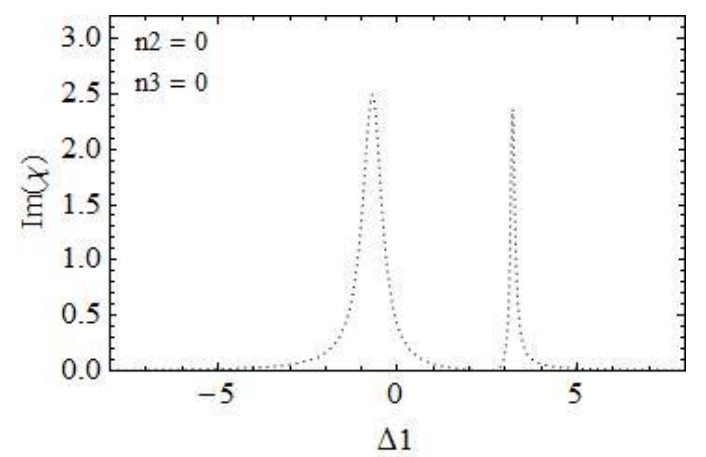

Fig. 6. Absorption spectrum of probe field versus detuning of an IYT atomic system in $n_{2}=n_{3}=0$. The parameters are similar to those chosen in Fig. 3. 


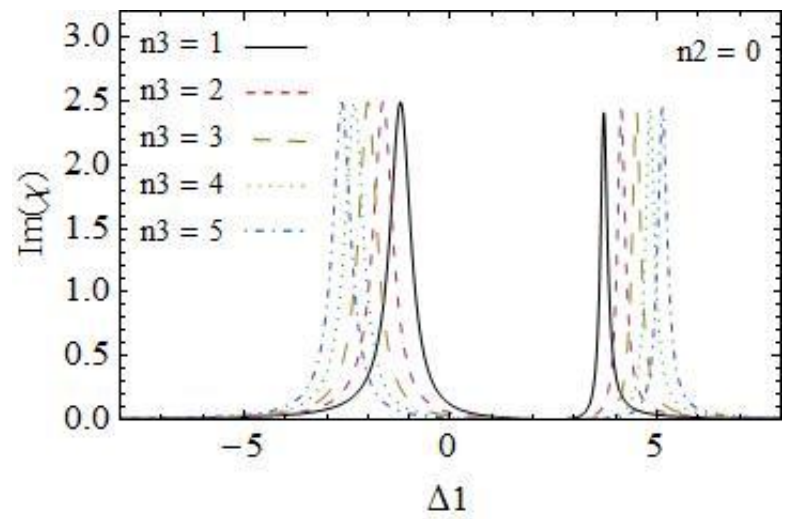

Fig. 7. Absorption spectra of the probe beam versus its detuning for an IYT atomic system interacting with the coupling photons; $n_{2}=0$ and $n_{3}=1,2,3,4,5$. The parameters are similar to those chosen in Fig. 3.

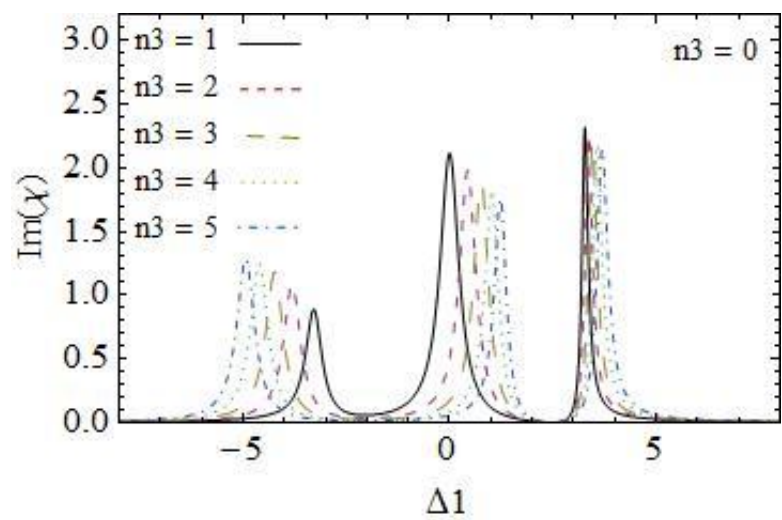

Fig. 8 Absorption spectra of the probe beam versus its detuning for an IYT atomic system interacting with the coupling photon; $n_{3}=0$ and $n_{2}=1,2,3,4,5$. The parameters are similar to those chosen in Fig. 3.

\section{III.A Photon Counting Method WITH FOUR-LEVEL IYT ATOMS}

Clearly, the detuning and amount of absorption for the peaks, in the absorption spectrum, are varied by the variation of each mode of coupling photons $n_{2}$ or $n_{3}$. It leads us to a photon counting method by a measurement of the absorption spectrum of the probe beam. This scheme is simpler than other photon counting methods because the number of photons (which is a microscopic quantity) is measured by the absorption spectrum of the probe field (which is a macroscopic quantity). It is also a non-demolition method for the weak probe fields.
In this section, we use analytical and numerical methods to obtain the numbers of two trapped coupling photons $n_{2}$ and $n_{3}$, simultaneously.

The detuning and amount of absorption spectrum for an IYT atom shown in Figs. 7 and 8 are depended on the number of coupling photons. There is a tight coupling between the measurement of a macroscopic quantity (absorption of probe beam) and measurement of a microscopic quantum mechanically computed quantity (the number of coupling photons). In this case, two different categories of absorption spectrum profiles of a probe beam of photons are investigated to obtain their corresponding number of coupling photons.

In the proposed experiment, a probe beam of individual photons is injected into an ensemble of trapped atoms, and its absorption is measured by a high sensitive single photodetector. By changing the detuning of the probe photons and performing the absorption measurements, we obtain an absorption spectrum similar to any one of the spectra in Figs. 7 and 8. In this section, the absorption spectra plotted in Figs. 7 and 8 are analyzed and the numbers of two modes of coupling photons are obtained, simultaneously.

Four different cases mentioned in Section two are applied to analyze the absorption spectra. The absorption spectra profiles have two categories: double peak and triple peak absorption spectrum. In the following, we present an analytical method to obtain the number of coupling photons for both the categories.

To derive an analytical relation for the number of photons in terms of defined parameters, the relation should be simpler by setting a resonance condition $\Delta_{2}=\Delta_{3}=0$. The detunings $\Delta_{2}$ and $\Delta_{3}$ should be controlled by a Stark effect of atomic levels due to a DC electric field. 
The first step of the photon counting procedure is the setting of detunings $\Delta_{2}$ and $\Delta_{3}$ to be in the resonance condition $\Delta_{2}=\Delta_{3}=0$. By these conditions, the absorption profile for both the categories transforms to a double peak (see Figs. 9(a) and 9(b)). In the second step, take derivative of the absorption spectrum with respect to the probe detuning $\Delta_{1}$, and set the result to zero. The difference between the detunings of absorption peaks $\delta_{\text {Inv. } \mathrm{Y}}=\left|\Delta_{\text {Peak } 1}-\Delta_{\text {Peak } 2}\right|$ is obtained as:

$\delta_{I n v . \mathrm{Y}}=2 \sqrt{g_{2}^{2} n_{2}+g_{3}^{2}\left(n_{3}+1\right)}$.

To derive Eq. (11), all decay rates are similar to those chosen in Fig. 3.

\section{A. Resonance Condition: First Category}

In the first category, it is easy to show that the number of coupling photons as $n_{2}=0$ (cases $b$ and d). If we apply it into (11), the number of next coupling photons is obtained as $n_{3}=\left(\delta_{I n v . \mathrm{Y}} / 2 g_{3}\right)^{2}-1$. Therefore the numbers of two coupling photons are obtained individually from the first category of the absorption spectrum. Indeed, our measurements need a calibration method.

To calibrate our setup, the cavities are initially evacuated from all coupling photons. In this case, the numbers of two coupling photons are zero and the absorption spectrum corresponding to the vacuum coupling fields is obtained. The difference between detunings of absorption peaks has a minimum value $\left(\delta_{I n v . \mathrm{Y}}\right)_{n_{2}=n_{3}=0}=2 g_{3}$. It is also a measurement method for $g_{3}$. Substitute $g_{3}$ into Eq. (11) to obtain the number of next coupling photons as:

\section{B. Resonance Condition: Second Category}

In the second category, the absorption spectrum contains a triplet absorption peak. In general, in this category, none of coupling photons are in the vacuum state. These spectra are more complicated than the first category and Eq. (11) is not enough to obtain the number of two modes coupling photons, individually.

To make the problem simpler, assume the resonance condition $\Delta_{2}=\Delta_{3}=0$. Triple peak absorption spectra transforms to a double peak by this condition. Figure 9 shows the different absorption peaks with respect to the different numbers of coupling photons. As shown in Fig.9, for a constant photon number $n_{2}\left(n_{3}\right)$, the amount of absorption peaks varies by changing the number of other coupling photons $n_{3}\left(n_{2}\right)$.

As can be seen in Fig. 9, each coupling photons number gives us a specific value of $\operatorname{Im}[\chi]$. So, distinct height of absorption peaks for different kinds of coupling photons causes the ability of photon counting in a four-level IYT atomic system. The maximum of absorption takes place at detuning

$$
\Delta_{1}=\sqrt{g_{2}^{2} n_{2}+g_{3}^{2}\left(n_{3}+1\right)},
$$

and its value is obtained from

$$
\chi_{b a}^{\prime \prime}=\operatorname{Im}\left(i\left[\left[\gamma_{1}+\gamma_{3}+i \Delta_{1}\right]+\frac{\left(n_{3}+1\right) g_{3}^{2}}{\gamma_{4}+i \Delta_{1}}+\frac{n_{2} g_{2}^{2}}{\gamma_{2}+i \Delta_{1}}\right]^{-1}\right) \text {. }
$$

Equations (13) and (14) are simultaneously solved to calculate the photon number of coupling fields, individually:

$n_{3}=\left(\left(\delta_{I n v . \mathrm{Y}}\right)_{n_{2}=0} /\left(\delta_{I n v . \mathrm{Y}}\right)_{n_{2}=n_{3}=0}\right)^{2}-1$.

$$
\begin{aligned}
& n_{2}=\frac{1}{2 A g_{2}^{4}\left(\gamma_{2}-\gamma_{4}\right)^{2}} \times \\
& {\left[\begin{array}{l}
-g_{2}^{2}\left(\gamma_{2}-\gamma_{4}\right)\left(\gamma_{2} \gamma_{4}-\Delta_{1}^{2}-2 A\left(\left(B^{2}+\left(\gamma_{1}+\gamma_{3}\right) \gamma_{4}\right) \gamma_{2}-\left(\gamma_{1}+\gamma_{2}+\gamma_{3}+\gamma_{4}\right) \Delta_{1}^{2}\right)\right) \\
+\sqrt{\left(-g_{2}^{4}\left(\gamma_{2}-\gamma_{4}\right)^{2}\left(\Delta_{1}^{2}\left(-\Delta_{1}^{2}-4 A \gamma_{4}\left(B^{2}+\left(\gamma_{1}+\gamma_{3}\right) \gamma_{4}-\Delta_{1}^{2}\right)+4 A^{2}\left(B^{2}+\left(\gamma_{1}+\gamma_{3}\right) \gamma_{4}-\Delta_{1}^{2}\right)^{2}\right)+2 \gamma_{2} \Delta_{1}^{2}\left(\gamma_{4}+4 A^{2}\left(\gamma_{1}+\gamma_{3}+\gamma_{4}\right)\left(B^{2}+\left(\gamma_{1}+\gamma_{3}\right) \gamma_{4}-\Delta_{1}^{2}\right)\right.\right.\right.}
\end{array}\right],}
\end{aligned}
$$


$n_{3}=-\frac{1}{2 A g_{2}^{2} g_{3}^{2}\left(\gamma_{2}-\gamma_{4}\right)^{2}} \times$
$\left[\begin{array}{l}-g_{2}^{2}\left(\gamma_{2}-\gamma_{4}\right)\left(\gamma_{2} \gamma_{4}-\Delta_{1}^{2}-2 A\left(g_{3}^{2}\left(\gamma_{2}-\gamma_{4}\right)+B^{2} \gamma_{4}+\gamma_{1} \gamma_{2} \gamma_{4}+\gamma_{2} \gamma_{3} \gamma_{4}-\gamma_{1} \Delta_{1}^{2}-\gamma_{2} \Delta_{1}^{2}-\gamma_{3} \Delta_{1}^{2}-\gamma_{4} \Delta_{1}^{2}\right)\right) \\ +\sqrt{\left.\begin{array}{l}\left(-g_{2}^{4}\left(\gamma_{2}-\gamma_{4}\right)^{2}\left(\Delta_{1}^{2}\left(-\Delta_{1}^{2}-4 A \gamma_{4}\left(B^{2}+\left(\gamma_{1}+\gamma_{3}\right) \gamma_{4}-\Delta_{1}^{2}\right)+4 A^{2}\left(B^{2}+\left(\gamma_{1}+\gamma_{3}\right) \gamma_{4}-\Delta_{1}^{2}\right)^{2}\right)+2 \gamma_{2} \Delta_{1}^{2}\left(\gamma_{4}+4 A^{2}\left(\gamma_{1}+\gamma_{3}+\gamma_{4}\right)\left(B^{2}+\left(\gamma_{1}+\gamma_{3}\right) \gamma_{4}-\Delta_{1}^{2}\right)\right.\right.\right. \\ \left.\left.\left.-2 A\left(B^{2}+2 \gamma_{4}\left(\gamma_{1}+\gamma_{3}\right)+\gamma_{4}^{2}-\Delta_{1}^{2}\right)\right)+\gamma_{2}^{2}\left(4 A\left(\gamma_{1}+\gamma_{3}\right)\left(-1+A\left(\gamma_{1}+\gamma_{3}\right)\right) \Delta_{1}^{2}+4 A\left(-1+2 A\left(\gamma_{1}+\gamma_{3}\right)\right) \gamma_{4} \Delta_{1}^{2}+\gamma_{4}^{2}\left(-1+4 A^{2} \Delta_{1}^{2}\right)\right)\right)\right)\end{array}\right]}\end{array}\right]$

where

$A=\frac{\chi_{b a}^{\prime \prime} \hbar \varepsilon_{0}}{N_{a} \wp_{b a}^{2}}$

is proportional to the maximum value of absorption and

$B=\sqrt{g_{2}^{2} n_{2}+g_{3}^{2}\left(n_{3}+1\right)}$,

is the value of its corresponding detuning. In Eqs. (15)-(18), the values of $\mathrm{A}$ and $\mathrm{B}$ are experimental data, which are obtained from the absorption spectrum given by the proposed setup.

The attention on the accuracy of absorption peak (and its detuning) measurement is important to obtain the exact number of coupling photons. Figure 10 illustrates the variation of absorption for neighbor peaks

$$
\delta\left(\operatorname{Im}\left[\chi_{n}\right]\right)=\operatorname{Im}\left[\chi_{n}\right]-\operatorname{Im}\left[\chi_{n+1}\right] \text {, }
$$

versus the coupling photon numbers. As one can see in Fig.16, the difference of absorption for neighbor peaks, $\delta\left(\operatorname{Im}\left[\chi_{n}\right]\right)$, decreases by increasing the number of coupling photons.

Therefore, the exact measurement for greater number of coupling photons needs more accuracy in the measurement of absorption peaks (and its detuning). So this model is suitable for a full quantum system or a few numbers of coupling photons. Development of our measurement method for the average number of photons, where the coupling fields are made of a superposition of number states, is an open problem.

\section{C.Non-resonance condition: Numerical Method}

In the previous subsection, an analytical method was introduced for the measurement of the number of coupling photons in the resonance condition. In this subsection, a numerical method is presented for the measurement of the number of coupling photons in a non-resonance condition.
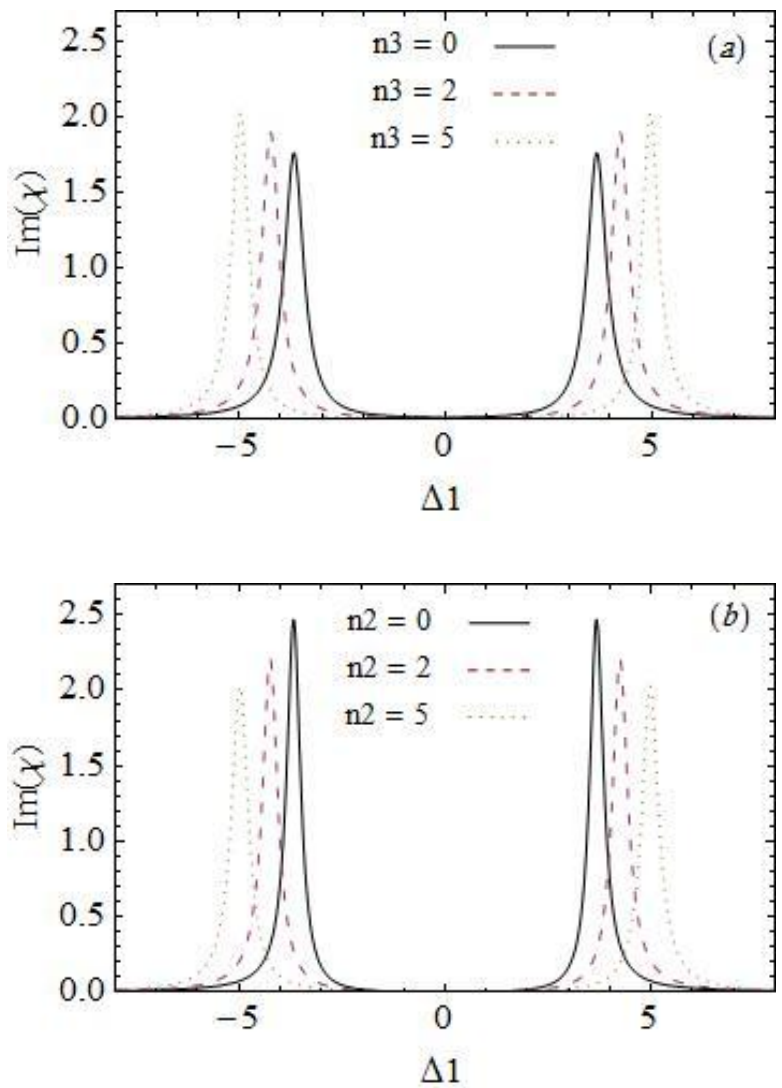

Fig. 9. Absorption spectra for an IYT atomic system in (a) constant $n_{2}=5$ and different $n_{3}=0,2,5$ and (b) constant $n_{3}=5$ and different $n_{2}=0,2,5$. The different numbers of coupling photons give us different values of absorption peaks. The parameters are similar to those chosen in Fig. 3.

In general, the absorption spectrum has a triple absorption peak. The differences between the detunings of absorption for edge and mid peaks are sufficient to individually determine the numbers of coupling photons. But the case of non-resonance condition is more complicated than the previous cases in the 
resonance condition. Therefore, we focus only on the numerical calculations of our method.
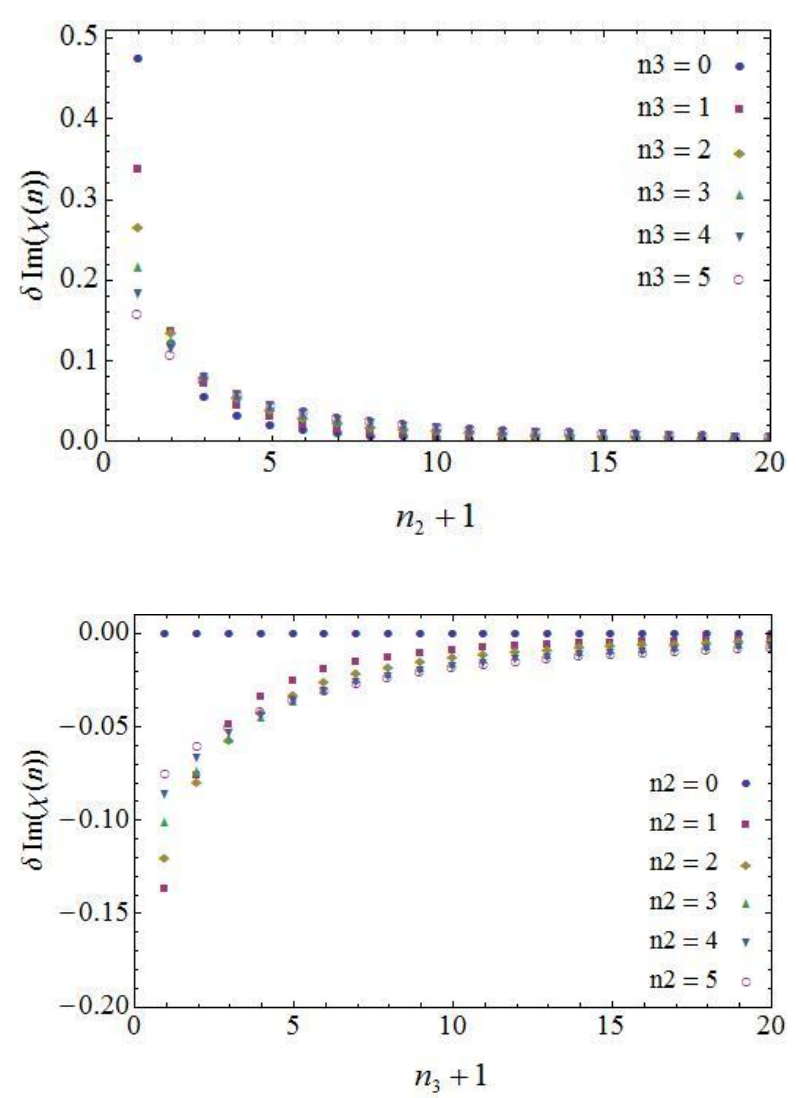

Fig. 10 The difference of absorption for neighbor peaks versus the different number of coupling photons, where the coupling fields are in the resonance condition. The parameters are similar to those chosen in Fig.3.

For our purposes, we define

$\delta_{+}=\left[\Delta_{1}\right]_{\text {RightMax }}-\left[\Delta_{1}\right]_{\text {IntervalMax }}$

and

$\delta_{-}=\left[\Delta_{1}\right]_{\text {IntervalMax }}-\left[\Delta_{1}\right]_{\text {LeftMax }}$.

where $\delta_{+}\left(\delta_{-}\right)$is the absolute difference between the detunings of right edge (left edge) and mid peaks. The value of $\delta_{+}$and $\delta_{-}$are obtained by the proposed setup in the nonresonance condition. Figure 11 indicates a couple of coupling photons $\left(n_{1}, n_{2}\right)$ in terms of $\delta_{+}, \delta_{-}$. Measurement of the difference between frequencies for edge(s) and mid peaks with a suitable accuracy gives us the number of coupling photons. Figure 11 shows the numerical relation between the measurement quantities $\delta_{+}$and $\delta_{-}$, and two kinds of coupling photons. Clearly, $\delta_{-}$is zero for a vacuum state of coupling field $v_{2}\left(n_{2}=0\right)$.

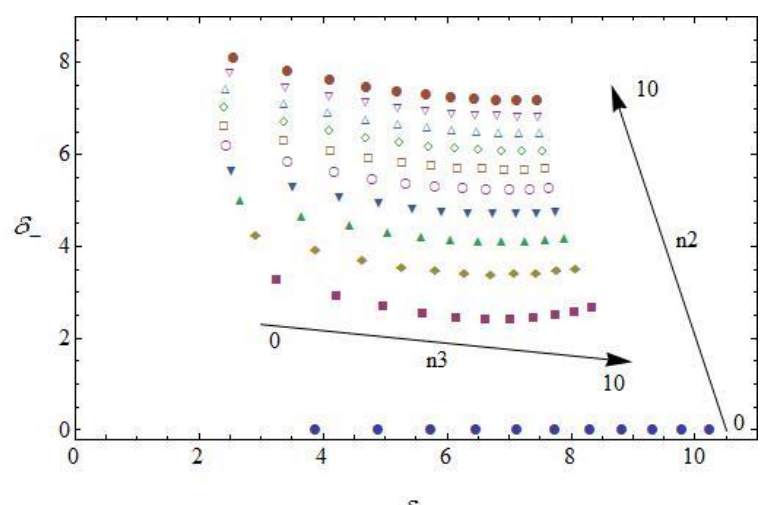

$\delta_{+}$

Fig. 11. Variation of $\delta_{+}$and $\delta_{-}$for different numbers of coupling fields $n_{2}=(0,1,2, \ldots 10)$ and $n_{3}=(0,1,2, \ldots 10)$ in a four level IYT atomic system, where the parameters are the same as those chosen in Fig. 3.

\section{IV.CONCLUSION}

In this article, we discussed four-level inverted Y-type (IYT) atomic systems trapped in a quantum cavity. The four-level IYT atoms are interacting with one kind of probe and two kinds of coupling field's photons. The absorption spectrum of the probe photons is derived from the imaginary part of susceptibility of atoms and plotted against its detuning.

The results are classified as follows:

In general, the four-level IYT atomic systems display triple peak absorption and two transparency windows in a non-resonance condition for coupling photons. Variation of the numbers of coupling photons and their detunings allows the possibility to control the width, shape and location of absorption peaks and transparency windows, efficiently.

The full quantum model of the four-level IYT atomic system indicates one window of transparency even where all coupling fields inside the cavity are in vacuum states. This transparency window is due to the effect of the vacuum state of the coupling filed. This 
phenomenon is observed only in the full quantum model, and not in the semi-classical model.

In the four-level IYT atomic system, with eliminating one of the coupling fields $v_{2}$, we cannot reach the absorption spectrum of corresponding three-level systems, because the effect of the vacuum state in the $v_{2}$ field.

The difference between the frequencies of the absorption peaks and also the values of absorption peaks are controllable by changing the number of coupling photons $\left(v_{2}\right.$ or $\left.v_{3}\right)$.

The properties of absorption spectrum profiles are discussed in different cases.

The results obtained in item 4 lead us to a nondemolition photon counting. Analytical relations between the number of coupling photons and different frequencies of absorption peaks and also the values of the absorption peaks are obtained in the resonance condition and weak field approximation.

Also in the non-resonance condition, the difference between the detunings of right (left) hand and mid peaks is applied to determine the number of two coupling photons numerically.

\section{REFERENCES}

[1] S. Deléglise, I. Dotsenko, C. Sayrin, J. Bernu, M. Brune, J-M. Raimond, and S.Haroche, "Reconstruction of non-classical cavity field states with snapshots of their decoherence," Nature, Vol. 455, pp. 510-514, 2008.

[2] S. Haroche, "Quantum Information with Atoms and Photons in a Cavity: Entanglement, Complementarity and Decoherence Studies," Physica Scripta, Vol. 2002, pp. 128-132, 2002.

[3] S. Khademi, Gh. Naeimi, S. Alipour, and Sh. Mirzaei, "An Exact Scheme for the EIT for a Three-level $\Lambda$-type Atom in a Quantum Cavity," Appl. Math. Information Sc. Vol. 9, No. 3, pp. 1225-1229, 2015.

[4] Gh. Naeimi, S. Khademi, and O. Heibati, "A Method for the Measurement of Photons Number and Squeezing Parameter in a
Quantum Cavity,” ISRN Optics, Vol. 2013, pp. 27195 (1-9), 2013.

[5] Gh. Naeimi, S. Alipour and S. Khademi, "A photon counting and a squeezing measurement method by the exact absorption and dispersion spectrum of $\Lambda$-type Atoms," Springer Plus, Vol. 5, pp. 1402-1414, 2016.

[6] S. Khademi, S. Alipour, and Gh. Naeimi, “ An exact solution method for upper-coupling three-level cascade atomic system interaction with a couple of quantized field in the steady state," J. Research Many Body Systems, Vol. 4, No. 7, pp. 1-9, 2014.

[7] V. A. Reshetov and E. N. Popov, "Single-atom quantum memory with degenerate atomic levels," J. Phys. B: At. Mol. Opt. Phys. Vol. 45, pp. 175501 (1-6), 2012.

[8] F. Bussieres, N. Sangouard, M. Afzelius, H. de Riedmatten, Ch. Simon and W. Tittel, "Prospective applications of optical quantum memories," J. Mod. Opt. Vol. 60, pp. 15191537, 2013.

[9] X.E. Maître, G. Hagley, C. Nogues, P. Wunderlich, M. Goy, Brune, J. M. Raimond, and S. Haroche "Quantum Memory with a Single Photon in a Cavity," Phys. Rev. Lett. Vol. 79, pp. 769-772, 1997.

[10]M. A. Nielsen and I.L. Chuang, Quantum computation and quantum information, Cambridge University Press, New York, NY: USA, 2000.

[11] M. Schlosser, S. Tichelmann, J. Kruse and G. Birkl, "Scalable Architecture for Quantum Information Processing with Atoms in Optical Micro-Structures," Quantum Information Proc. Vol. 10, No. 6, pp. 907-924, 2011.

[12] G.W. Lin, X.B. Zou, X.M. Lin, and G.C. Guo, "Scalable, high-speed one-way quantum computer in coupled-cavity arrays," Appl. Phys. Lett. Vol. 95, pp. 224102 (1-3), 2009.

[13]P. Kok, W. J. Munro, K. Nemoto, T.C. Ralph, J.P. Dowling, and G.J. Milburn, "Linear optical quantum computing with photonic qubit," Rev. Mod. Phys. Vol. 79, No.1, pp. 135-174, 2007.

[14] N.H. Abdel-Wahab and L. Thabet, "The influence of the relative phase on the interaction between a single mode cavity field and a four-level N-type atom," Eur. Phys. J. Plus, Vol. 128, No. 52, pp. 13052-13060, 2013. 
[15] J. Evers, "Phase-dependent interference mechanisms in a three-level $\Lambda$ system driven by a quantized laser field," J. Mod. Opt. Vol. 52, pp. 2699-2712, 2005.

[16]M.O. Scully and M.S. Zubairy, Quantum optics, Cambridge: Cambridge University Press, 1997.

[17]C.C. Gray, P.L. Knight, Introductory Quantum Optics, Cambridge, New York, 2005.

[18] M.J. Faghihi and M.K. Tavassoly, "Dynamics of entropy and nonclassical properties of the state of a $\Lambda$-type three-level atom interacting with a single-mode cavity field with intensitydependent coupling in a Kerr medium," J. Phys. B: At. Mol. Opt. Phys. Vol. 45, No.3, pp. 035502 (1-14), 2012.

[19] M.J. Faghihi, M.K. Tavassoly, "Number-phase entropic squeezing and nonclassical properties of a three-level atom interacting with a twomode field: Intensity-dependent coupling, deformed Kerr medium and detuning effects," J. Opt. Soc. Am. B, Vol. 30, No.11, pp. 28102818, 2013.

[20] O. Kocharovskaya and Y.I. Khanin, "Coherent amplification of an ultrashort pulse in a threelevel medium without a population inversion," JETP Lett. Vol. 48, No.11, pp. 630-634, 1988.

[21] S.E. Harris, J.E. Field, and A. Imamoglu, "Nonlinear optical processes using electromagnetically induced transparency," Phys. Rev. Lett. Vol. 64, pp. 1107-1110, 1990.

[22] S.E. Harris, "Electromagnetically Induced Transparency," Phys. Today, Vol. 50, No. 7, pp. 36-42, 1997.

[23] A.M. Akulshin, S. Barreiro, and A. Lezama, "Electromagnetically induced absorption and transparency due to resonant two-field excitation of quasi degenerate levels in $\mathrm{Rb}$ vapor," Phys. Rev. A, Vol. 57, pp. 2996-3002, 1998.

[24] Y. Zhang, Zh. Wu, X. Yao, Zh. Zhang, H. Chen, H. Zhang, and Y. Zhang, "Controlling multi-wave mixing signals via photonic band gap of electromagnetically induced absorption grating in atomic media," Opt. Express, Vol. 21, No. 24, pp. 29338-29349, 2013.

[25] M. Marthaler, Y. Utsumi, D.S. Golubev, A. Shnirman, and Gerd Schön, "Lasing without Inversion in Circuit Quantum
Electrodynamics," Phys. Rev. Lett. Vol. 107, No. 9, pp. 093901 (1-5), 2011.

[26] M. O. Scully, S. Y. Zhu and H. Fearn, "Lasing without inversion," Zeitschrift Physik D: Atoms, Molecules and Clusters, Vol. 22, No. 2, pp. 471-481, 1992.

[27] S.H. Autler and C.H. Townes, "Stark effect in rapidly varying fields," Phys. Rev. Vol. 100, pp. 703-722, 1955.

[28]E.H. Ahmed, J. Huennekens, T. Kirova, J. Qi, and A. M. Lyyra, "The Autler-Townes Effect in Molecules: Observations, Theory, and Applications," Adv. Atom., Molec. Opt. Phys. Vol. 61, pp. 467-514, 2012.

[29] T.Y. Abi-Salloum, "Electromagnetically induced transparency and Autler-Townes splitting: Two similar but distinct phenomena in two categories of three-level atomic systems," Phys. Rev. A, Vol. 81, No. 5, pp. 053836 (1-6), 2010.

[30]M. Sahrai, S. Aas, and M. Mahmoudi, "Subluminal to superluminal pulse propagation through one-dimensional photonic crystals with a three-level atomic defect layer," Eur. Phys. J. B, Vol. 78, No. 1, pp. 5158, 2010.

[31] M. Mahmoudi, S. W. Rabiei, L. Safari, and M. Sahrai, "Gain-assisted superluminal light propagation via incoherent pump field," Laser Phys. Vol. 19, No. 7, pp. 1428-1433. 2009.

[32]M. Mahmoudi, S. W. Rabiei, L. Ebrahimi Zohravi and M. Sahrai, "Absorption free superluminal light propagation in a three level pump-probe system," Opt. Commun. Vol. 281, No. 18, pp.4681-4686, 2008.

[33] M. Mahmoudi, S.M. Mousavi, and M. Sahrai, "Controlling the optical bistability via interacting dark-state resonances," Eur. Phys. J. D, Vol. 57, No. 2, pp. 241-246, 2010.

[34] T. G. Walker and M. Saffman, "Entanglement of Two Atoms Using Rydberg Blockade," Adv. At. Mol. Opt. Phys, Vol. 61, 2012.

[35] A. Joshi and M. Xiao, "Electromagnetically induced transparency and its dispersion properties in a four-level inverted-Y atomic system," Phys. Lett. A, Vol. 317, No. 5-6, pp. 370-377, 2003.

[36]D.A. Steck, Rubidium 85 D Line Data, available: online at http://steck.us/alkalidata. 


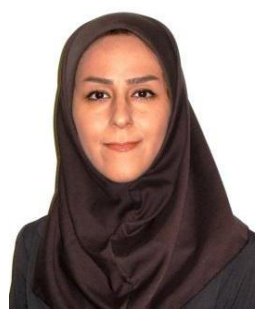

Samira Alipour received her BSc. and MSc. degrees in Atomic Physics from K.N. Toosi University of Technology and Amir-kabir University of Technology, Tehran, Iran, respectively. Now, she is a $\mathrm{PhD}$ candidate in Quantum Optics at University of Zanjan, Zanjan, Iran. Her research interests are quantum optics, laser physics, nonlinear optics, and photonic band gap materials.

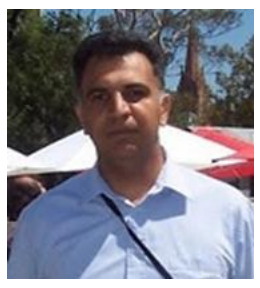

Siamak Khademi was born in 1965, in Shiraz, the capital city of Fars Province. He received his BSc. degree in applied physics from Isfahan University, Isfahan, Iran, and his MSc. and $\mathrm{PhD}$ degrees in (Atomic and Molecular) physics from Shiraz University, Shiraz, Iran. His research interests are in the areas of fundamental quantum mechanics, quantum phase space, quantum information, entangled systems, quantum optics, and physics education. He has been working at the Department of Physics of the University of Zanjan, Iran, since 2001. 